\title{
A Broadcast Vehicle to Vehicle Communication System in Railway Environments
}

\author{
Cristina Rico García \\ Institute of Communications \\ and Navigation \\ German Aerospace Center \\ Oberpfaffenhofen, 82234 \\ Weßling \\ cristina.ricogarcia@dlr.de
}

\author{
Thomas Strang \\ Institute of Communications \\ and Navigation \\ German Aerospace Center \\ Oberpfaffenhofen, 82234 \\ Weßling \\ thomas.strang@dlr.de
}

\author{
Andreas Lehner \\ Institute of Communications \\ and Navigation \\ German Aerospace Center \\ Oberpfaffenhofen, 82234 \\ Weßling \\ andreas.lehner@dlr.de
}

\begin{abstract}
Current statistics in railway transportation systems show that the number of accidents is still too high, despite of huge investments in infrastructure-based safety systems. We show that a infrastructure-less cross-layer train-to-train communication system exploiting all characteristics of a pervasive computing system, like direct communication in mobile adhoc networks (MANETs), exchange of location and other relevant context information provided by multiple sensors in the trains, can reveal hazardous situations. While maritime and air transport are already benefiting from collision avoidance applications based on infrastructure-less communications and similar applications will be available for road users built on top of car-to-car communications soon, an equivalent for rail transport systems is not existing yet. In order to design such a system, a six phase work approach is conducted: Preliminary analysis and selection of an adequate frequency band, characterization of the propagation channel, MAC layer design, physical layer and finally verification of the system. During this process, context information provided by navigation systems and other sensors, e.g. position, time and speed are utilized to improve the communication.
\end{abstract}

\section{INTRODUCTION}

Current statistics of the International Union of Railways (UIC) show, that there are three significant train accidents in Europe every day [2], despite millions of Euros which have been invested in trackside and in-train safety equipment. In order to increase safety in railway traffic, some countries are partially installing control systems, mainly centrally managed ones, specially the Automatic Train Control (ATC), where the trains are monitored by devices located along the rail. These devices send the collected information to an operation center that can pass specific instructions to the train. A European ATC standard, European Train Control Sys-

ISVCS 2008 July 22 - 24, 2008, Dublin, Ireland ISBN: 978-963-9799-27-1 tem (ETCS), is intended to replace the various European ATC systems, in order to protect international train traffic. However, according to estimations of the German railway company "Deutsche Bahn" (DB), it could take up to 20 years and cost up to 8 billion Euro to introduce ETCS right across Europe [1]. Furthermore, only the operation center has an overall overview of the traffic situation, and a train driver could only be warned against hypothetical collisions if the operation center decides so.

While maritime, air, and road transport have a vehicle integrated collision avoidance system available or in the development phase, we find no satisfactory solution of this type of technology in railway transportation.

Therefore it is necessary to develop a system that will allow the train conductors to have an up-to-date accurate knowledge of the traffic situation in the vicinity and act in consequence. The system is intended to not rely on components in the infrastructure, this way substantially reducing its rollout- and maintenance costs, as well as inherently providing a migration strategy. The basic idea is to communicate relevant own context information to all other nearby trains. In this particular case, each trains has to calculate its own position and movement vector and broadcast this information as well as additional data like vehicle dimensions to all other trains in the area. Thus, the driver's cabin could be equipped with a display showing the position of the other vehicles in the region. Computer analysis of all received context information, the own position and movement vector and an electronic track map detects possible collisions, displaying an alert signal, and advising the driver of the most convenient strategy to follow in order to avoid the danger. The system can take into account different danger sources, like advancing trains or road vehicles or obstacles, and classify them according to a specific scale.

\section{PROBLEM DOMAIN AND EXPECTED CONTRIBUTIONS}

The aim of our work is the design, development, simulation, optimization and verification of a communication system that will allow the transmission of messages of a railway collision avoidance system. We need to consider the physical constraints of the application in order to infer the communication parameters as shown in Figure 1. In particular the distance the trains need to brake, their speed, the number of trains in a network and the characteristics of the propagation 
channel will delimit the communication system features.

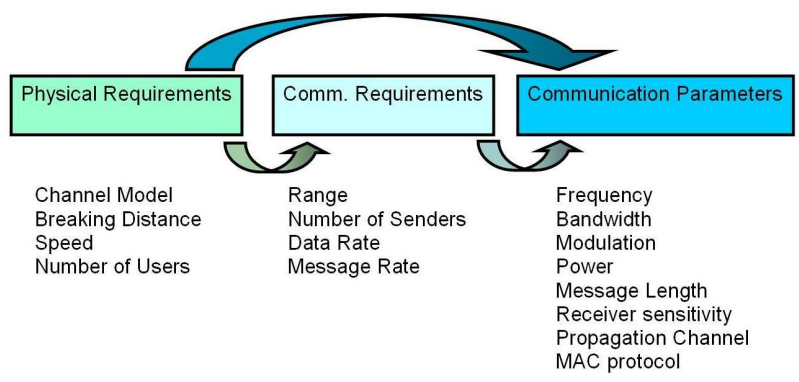

Figure 1: Relationship between physical requirements, and communication parameters.

The following challenges arise designing the system:

- No infrastructure can be used:

Wireless communication introduces great complexity in any communication system. Many of these systems, especially when the range is a constraint, make use of infrastructure like repeaters in order to extend the network range. However, this strategy has a negative financial impact. Therefore, our system is infrastructureless and we have to deal with a careful design of the communication parameters, particulary with the physical layer, in order to guarantee for an adequate range.

- The system will run in regional railway networks: As major efforts in railway safety are being concentrated on high speed lines, regional networks are a forgotten area nowadays. However, this kind of lines offers an interesting perspective and adds major difficulties to the communication system. In contrast to high speed lines, regional railway networks are characterized by sharp and profuse number of curves. Therefore, it can be expected a propagation channel with high fading and multipath, which has a severe impact on the communication level. The available channel models for railway networks are focused on high speed lines. Thus it is necessary to develop a suitable channel model.

- Transmissions are broadcast: The messages sent by a train should be received by all the neighbors in the area with as little delay as possible. Thus broadcast is the most suitable strategy. The resulting communication network is a broadcast mobile ad-hoc network (MANET) characterized by high sender density and large speed. Despite, a reliable MAC layer for these kind of networks is nowadays a major concern and interest of investigation: no satisfactory solution has been found yet.

- The trains send context information regarding themselves provided by a GNSS unit and other sensors: Since the system possesses an onboard GNSS unit, the available information, e.g. timing, position, speed and direction, can be used in order to optimize the communication in several levels like the Physical and MAC layers. Consequently, the communication layers will be orientation aware. 


\section{RELATED WORK}

Safety systems in railway transportation still rely on old optical or mechanical mechanisms like semaphores. However, there is a new safety system for railway transportation based on GSM that has been recently developed called GSM$\mathrm{R}$. The fact, that it relies strongly on infrastructure makes its deployment extremely expensive. Therefore, GSM-R is mainly focused on high speed lines leaving regional networks unprotected while the deployment will be slow and costly. Furthermore, since GSM-R is a centralized system, i.e communications pass through a central station, it will be more susceptible to errors. In contrast, we will propose a collision avoidance system that overcomes all these problems. It is infrastructure-less and mainly focused on regional networks.

There are a number of approaches of infrastructure-less collision avoidance systems for other transportation systems, e.g. Automatic Identification System (AIS) [3] for Maritime, Automatic Dependance Surveillance (ADS-B) [4] for air, and Car2Car [5] for road transportation. The surveillance application of these systems share some characteristics: they are broadcast, distributed and in their application level they use the information provided by a GNSS system. However, due to the differences in the environmental characteristics and requirements of the transportation systems they are generally not applicable to the railway transportation. The manoeuvring possibilities are in railway transportation considerably reduced and thus, the reaction options are mainly limited to brake the train. The potential speed of the trains, combined with the reduced reaction capabilities and the geographical proximity between adjacent rails, introduces a high accuracy requirement on position determination. The high density of vehicles on roads that allows network extension through multihop cannot be assumed in railway transport. Therefore, communication layers above the Medium Access will not be considered any further. The network that the trains build can be regarded as very dynamical, since they stay only shortly in the range of other trains in the area. This adds major difficulties in the MAC layer. Particularly the differences in the physical layer are more palpable. Air and maritime transportation require large ranges. However, the channel characteristics are much less restrictive than in railway environment. On the other hand, the channel in road transportation is comparable to the one in railway. Nonetheless, its necessary range is much shorter. All these factors lead to the conclusion that railway transportation imposes the highest challenges [8].

\section{DESIGN OF AN ORIENTATION AWARE RAILROAD COLLISION AVOIDANCE SYSTEM}

\subsection{Preliminary analysis and selection of an adequate transmission band}

The aim of the preliminary analysis is to point out the parameters that should be designed. It has to be explained how to infer these values from the physical characteristics of a railway transportation system. Furthermore, an approximate value of these communication parameters must be given, as well as the key aspects and influence that the parameters will produce in the overall system will be noted. Since the frequency is a key aspect that will condition the

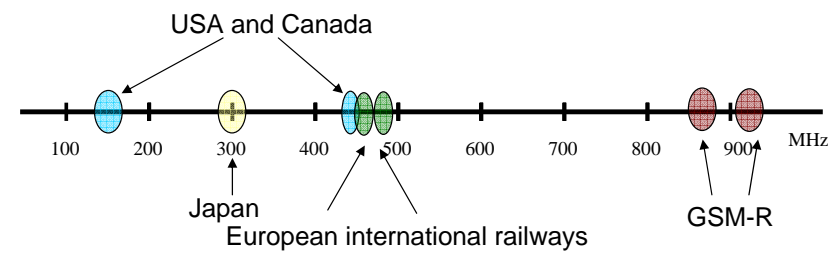

Figure 2: Worldwide distribution of frequency bands for railway applications

design of the whole system, it should be the first parameter to be chosen.

Taking into account all circumstances of the system itself and the current availabilities (see the current frequency occupations in Figure 2) we picked the $400 \mathrm{MHz}$ band for our system and many more characteristic parameters that can be found in [8] jointly with the reasons of their deduction.

\subsection{Characterization of the Propagation Chan- nel}

In order to optimize the communication link, it is necessary to approach the design attending the specific characteristics of the channel. Since one of the most critical requirements is the communication range [8], the channel model should be chosen carefully. A deterministic approach to establish the parameters of the propagation channel is not feasible due to the highly dynamic railway channel characteristics that can change the instantaneous amplitude very rapidly.

Relatively little work has been undertaken on characterizing the propagation environment for railways. Most of the available work deals with GSM-R [9], which uses a proprietary frequency band $(876-880 \mathrm{MHz}$ uplink, 921-925 $\mathrm{MHz}$ downlink). However, in contrast to GSM-R we cannot assume for instance LOS, as our system will work on regional networks and not only on high speed lines. There have been undertaken as well some analysis of deterministic channel models in the $25 \mathrm{GHz}$ band [10] and in the $5 \mathrm{GHz}$ band [11]. In general, all this research is done for high speed lines. On the other hand general propagation prediction models for different terrain profiles, like Hata-Okumura, Ibrahim and Parson are widely used when planning a terrestrial system [12].

Our characterization of the propagation channel (like in Figure 3 , see [7]) is focused on a defined frequency band and takes into account:

\section{Path loss:}

Accurate prediction methods are needed to determine the parameters the system which has to provide efficient and reliable coverage. Factors that influence the signal strength are buildings and other man made obstacles, which produce multipath. On the other hand, trees and other vegetation cause shadowing, scattering and absorption.

2. Doppler: 
It is well known that the relative motion of transmitter and receiver produces an apparent change in frequency. This is the Doppler shift. The maximum Doppler frequency shift occurs when the communicating trains run in opposite direction on a straight line. As a result of the existence of many scatters coming from different directions, the apparent frequency shift will be diverse for each scatter. Hence, a Doppler spectrum is shaped.

\section{Fading:}

Due to changes in the environment, fluctuations of various tens of decibels around this median value are feasible. There is a variation in the median signal as the train moves from place to place caused by large-scale variations in the terrain profile along the path due to changes in the nature of the local topography. This is the slow fading, characterized by a log-normal distribution. Fast fading are variations caused by multipath propagation in the immediate vicinity. When good visibility exists, LOS is possible and the channel is Ricean, in the other case the channel is Rayleigh.

4. Delay Spread:

This parameter describes the multipath effect over the bandwidth of the signals. If the transmitted signal bandwidth is sufficiently small, so that all the frequencies behave similarly, then the channel has flat fading. In this case, the delay of the paths is spread inside the transmitted symbol. When the delay spread is greater than the symbol period, the channel exhibits frequency-selective fading and yields intersymbol interference (ISI) which produces severe distortion of the signal. For that reason, the delay spread delimits the coherence bandwidth, the spectral area were the transmitted signal is not severely distorted.

5. Noise and interference:

Although this parameter is usually not included in the Channel models, it is an important value, since it indicates how good the receiver should be.
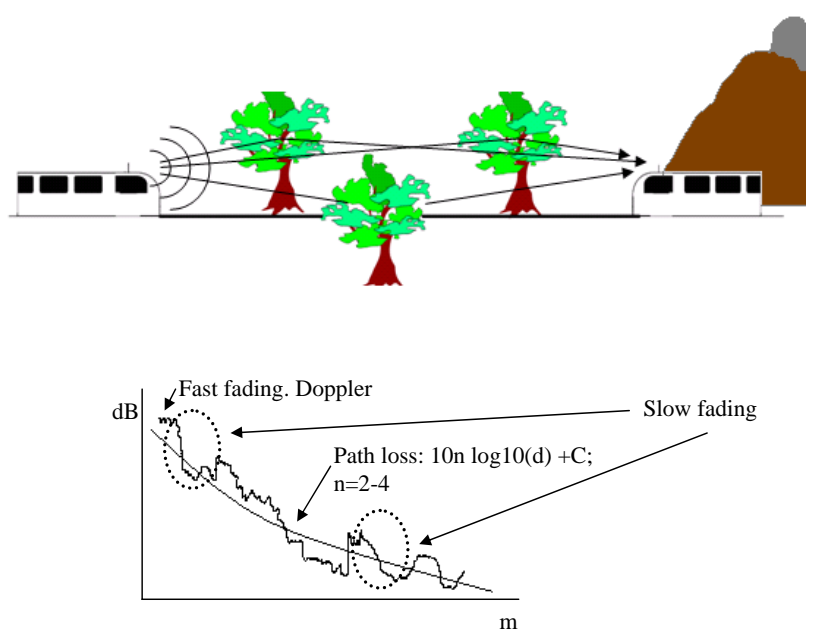

Figure 3: Complete characterization of a channel

\subsection{MAC Layer}

The MAC layer has to be carefully designed as it defines the throughput of the system. Since there is no upper layer to manage packet collisions, the MAC layer should avoid packet collisions or ensure a suitable low level of collision rate for a surveillance application. At this point appropriate message length and message rate should be selected in order to complete the specifications of the MAC layer.

The simplest protocol that can be used is the well known ALOHA protocol [13] where no control is used. However due to its low throughput it is only applicable in low density ad-hoc networks. Another one of the earliest mechanism adopted was the CSMA (Carrier Sense Medium Access) protocol [14]. Nonetheless it introduces the hidden terminal and exposed terminal problem. Thus, a variety of more complex MAC protocols have been proposed for mobile ad-hoc networks (MANETs). Some of these general protocols include the Multiple Access with Collision Avoidance (MACA) [15], Media Access Protocol for Wireless LAN's (MACAW) [16], Floor Acquisition Multiple Access (FAMA) [17] and the IEEE 802.11 MAC protocol [18].

Unfortunately, these protocols are not suited for broadcast MANETs as they use handshake techniques that are not applicable for broadcast to unknown communication partners. Many MAC broadcast protocols for MANETs have been proposed in the literature. They can be classified in two groups:

- Improved versions of the widely accepted IEEE 802.11 MAC protocols, all relying on handshake. They include BSMA [20], BMW [21], BMMM [22], LAMM [22]

- Innovative MAC protocols related to TDMA or CDMA. In this group we can find ADHOCMAC [23], FivePhase Reservation Protocol (FPRP) [19], Self Organized Time Division Multiple Access (SOTDMA) [24], CATA [25], ABROAD [26], RBRP [27] and SNDR [28].

Another major concern and interest of investigations are complex and very extended MANETs, characterized by high sender density and large speed, like the system we aim to design. Unfortunately, the proposed broadcast MANETs MAC protocols are not suited for this kind of networks. Those based on IEEE 802.11 MAC use handshake techniques in order to recognize possible packet collisions and retransmit again the messages. On the other hand the innovative ones assume quite static networks, a prior knowledge of the number of receivers and in some cases the hidden and exposed terminal problem are not solved. Since GNSS is present in our system, the information given by the GNSS system (location, direction, speed, and precise timing) can be utilized by the MAC layer. A number of MAC protocols based on location awareness have been proposed, like GRID [29] and TPCPC [30]. However, these protocols are focused on unicast communication. Moreover, they rely on a handshake protocol, and therefore, there might be collisions during the contention.

That's why we designed a new MAC layer protocol called Cell-based Orientation-Aware MANET Broadcast (COMB) 


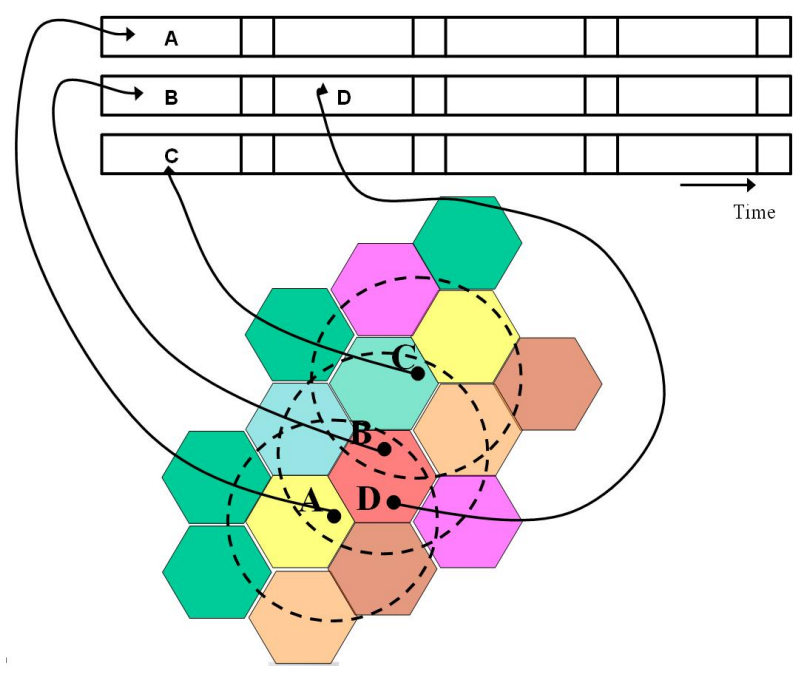

Figure 4: Cell-based Orientation-Aware MANET Broadcast (COMB) Protocol. A MAC layer protocol suitable for railroad purposes.

Protocol [6] that is able to overcome all the afore mentioned shortcomings by a combination of CDMA and SOTDMA, see also Figure 4.

Hence a simulation environment where the MAC layers for train to train communication can be tested should be developed. In this environment the suitability of traditional, recently published layers and our newly designed COMB protocol will be investigated, compared and optimized.

\subsection{Physical Layer}

In the PHY layer such important parameters as modulation, bandwidth, power, channel coding etc. are specified. In order to design it, these parameters must provide the required range and data rate which are given by the propagation channel characteristics, train speed, and necessary breaking distance of the trains upon actual circumstances. The range is defined as the maximum distance between transmitter and receiver so that a sufficient signal to noise ratio is guaranteed at the input of the receiver. The frequency influences the decrease experimented by the signal level due to distance when an omnidirectional antenna is used. A higher transmitted power guarantees with a directly proportional relation a larger range. On the other hand, the influence introduced by the propagation channel and the protection given by the utilized digital modulation scheme define the noise level at the receiver. The upper bound of the data rate is related with the Shannon-Hartley theorem: "The amount of information an electromagnetic wave can carry is related to its bandwidth. The signal to noise ratio is related with the bit error rate (BER), i.e, a low signal to noise ratio leads to a high BER, thus decreasing the data rate. Otherwise, high spectral efficient digital modulation increases the data rate automatically due to larger bits per symbol values. However, high spectral efficiency implies a larger probability of error for the same noise level at the input of the demodulator. Despite adding bits, channel coding might correct the errors in the signal due to noise, leading to an increment in the data rate.
All these aspects should be carefully studied, designed and finally a simulation environment, that will allow the test of the developed physical layer, will be built, where simulation of the resulting design in combination with MAC layer will be carried out. Furthermore, consecutive refinements of the system should be preformed.

\subsection{Verification}

The last phase takes into consideration the suitability of the system under real circumstances, its performance and clarification of open issues/inconsistencies regarding the theoretical channel model.

\section{CONCLUSION}

In this paper we presented the need for a infrastructureless orientation-aware broadcast railroad collision avoidance system. We sketched the ideas that lead to a solution of the shortcomings of available systems or the adaptation of existing systems to the challenges of a railroad environment.

The steps to the completion of the system design are detailed above and partially already performed as can be seen in further publications of the German research project RCAS.

\section{REFERENCES}

[1] Gerhard Schmid, "19 Risk management in rail transportation", Casualty Risk Consulting Information for insurers, Münchener Rückversicherungs-Gesellschaft, Germany, 2006.

[2] Safety Database Project Team (UIC-SDB), "State of the Art", The UIC Safety Data Base (UIC-SDB), Paris, 2006.

[3] AIS official website, http://www.navcen.uscg.gov/enav/ais/default.html

[4] International Standards and recommended practices. Aeronautical Telecommunications. Annex 10, To the convention on international civil aviation. Volume IV Surveillance Radar and collision avoidance systems. 1 March 2005.

[5] Timo Kosch "Technical Concept and prerequisites of car-to-car communication" Technical report, BMW Group Research and Technology, 2002.

[6] Cristina Rico García, Andreas Lehner and Thomas Strang, "COMB: Cell based Orientation aware MANET Broadcast MAC layer", to be published, 2008

[7] Cristina Rico García, Thomas Strang, Andreas Lehner and Korbinian Frank, "Channel Model for Train to Train Communication using the $400 \mathrm{MHz}$ Band", 67th Vehicular Technology Conference (VTC2008-Spring), Marina Bay, Singapore, 2008.

[8] Cristina Rico García, Andreas Lehner, Thomas Strang and Matthias Röckl, "Comparison of Collision Avoidance Systems and Applicability to Rail Transport", 7th International Conference on Inteligent Transportation Systems Telecommunication (ITST 2007), Sophia Antipolis, France, 6-8 June 2007.

[9] Farrokh Abrishamkar and James Irvine, "Comparison of Current Solutions for the Provision of Voice Services to Passengers on High Speed Trains", Vehicular Technology Conference (VTC 2000), Tokyo, Japan, 15-18 May, 2000. 
[10] Tetsunori Hattori, Kohshi Abe and Ken-ichi Abe, "Analyses of Propagation Characteristics in Future Railway Communication Systems Using 25GHz Band Radio", 49th. IEEE Vehicular Technology Conference (VTC 1999), Houston, Texas, USA, 16-19 May, 1999.

[11] Sandra Knözer, Michael A. Baldauf, Thomas Fügen and Werner Wiesbeck, "Channel Characterisation for an OFDM-MISO Train Communication System", 6th International Conference on Inteligent Transportation Systems Telecommunication (ITST 2006), Chengdu, China, 21-23 June, 2006.

[12] Tapan k. Sarkar, Zhong Ji, Kyungjung Kim, Abdellatif Medouri and Magdalena Salazar-Palma, "A Survey of Various Propagation Models for Mobile Communication", IEEE Antenna and Propagation Magazine, Vol 45, No 3, June 2003.

[13] N. Abramson, "The ALOHA System-Another alternative fo rcomputer communications", 1970 Fall Joint Comput. Conf., AFIPS Press, vol37, pp. 281-285, 1970.

[14] John Jubin and Janet D. Tornow, "The DARPA Packet Radio Network Protocols", Proceedings of the IEEE, January 1987.

[15] P. Karn, "MACA-A New Channel Access Method for Packet Radio", Amateur radio 9th Computer Networking Conference, ARRL, 1990.

[16] R.Bargrodia, A. Demers, S.Shenker, and L. Zhang, "MACAW: A Media Access Protocol for Wireless LAN's" ACM SIGCOMM, 1994.

[17] C. Fullmer and J.J Garcia-Luna-Aceves, "Floor Acquisition Multiple Access (FAMA) for packet radio networks", ACM SIGCOMM, Cambridge, October 1995.

[18] Editors of IEEE 802,11, Wireless LAN Medium Access Control (MAC and Physical Layer (PHY) specifications, Draft Standard IEEE 802.11, 1997.

[19] Chenxi Zhu and M. Scott Corson, "A Five-Phase Reservation Protocol (FPRP) for Mobile Ad-Hoc Networks" Proc. INFORCOM'98, 1998.

[20] K. Tang and M. Gerla, "Random access MAC for efficient broadcast support in ad hoc networks", Proceedings of the IEEE Wireless Communications and Networking Conference (WCNC), pp. 454-459, September 2000.

[21] K. Tang and M. Gerla,"MAC reliable broadcast in ad hoc networks", Proceedings of the IEEE Military Communications Conference (MILCOM), pp. 1008-1013, October 2001.

[22] M. T. Sun, L. Huang, A. Arona, and T. H. Lai "Reliable MAC layer multicast in IEEE 802.11 wireless networks", Proceedings of the IEEE International Conference on Parallel Processing (ICCP), pp. 527-536, August 2002.

[23] F. Borgonovo et al. "ADHOC MAC: a new, flexible and reliable MAC architecture for ad-hoc networks" IEEE Wireless Communications and Networking Vol. 2, WCNC 2003.

[24] Høakam Lans, Saltsjöbaden, "Position Indicating System" United States Patent, Patent Number: 5506587, Apr. 9, 1996.

[25] Z. Tang and J.J. Garcia-Luna-Aceves, "A protocol for topology-dependent transmission scheduling in wireles networks" Proceedings of IEEE WCNC'99, pp. 1333-1337, September 1999.

[26] I. Chlamtac, A. D. Myers, V.R. Syrotiuk, and G. Zaruba, "An adaptive medium access contorl (mac) protocol for reliable broadcast in wireless networks" Proceedings of IEEE ICC'00, June 2000.

[27] Mahesh K. Marina, George D. Kndylis, Ulas C. Kozat, "A Robust Broadcast Reservation Protocol for Mobile Ad Hoc Networks" Proceedings of the IEEE International Conference on Communications (ICC), vol. 1, pp. 878-885, June 2001.

[28] Zhijun Cai and Mi Lu, "SNDR: A New Medium Access Control for Multi-channel Ad Hoc Networks", Proceedings of the Vehicular Technology Conference (VTC), Tokyo 2000.

[29] Yu-Chee Tseng, Chih-Min Chao, Shih-Lin Wu, Jang-Ping Sheu, "Dynamic channel allocation with location awareness for multi-hop mobile ad hoc neworks" Computer Communications, Elsevier, 25, pp. 676-688, 2002.

[30] Lili Zhang, Boon-Hee Soong, Wendong Xiao, "A New Multi-Channel MAC Protocol for Ad Hoc Networks Based on Two-phase Coding with Power Control (TPCPC)" Fourth International Conference on Information, Communications \& Signal Processing and Fourth IEEE Pacific-Rim Conference On Multimedia(ICICS-PCM 2003), Singapore, 15-18 December 2003. 